\begin{tabular}{|c|c|c|c|}
\hline$\underbrace{\text { INTERNATIONAL }}_{\text {INESEG }}$ & $\begin{array}{r}\text { International } \\
\text { Res } \\
w w v\end{array}$ & $\begin{array}{l}\text { ournal of Health Services } \\
\text { arch and Policy } \\
\text { lergipark.org.tr/ijhsrp }\end{array}$ & \\
\hline $\begin{array}{l}\text { SCIENCE AND } \\
\text { EDUCATION GROUP }\end{array}$ & e-ISSN: 2602-3482 & DOI: 10.33457/ijhsrp.670597 & IJHSRP \\
\hline
\end{tabular}

Research Article

\title{
CAPABILITY MODEL AND COMPETENCE MEASURING FOR SMART HOSPITAL SYSTEM: AN ANALYSIS FOR TURKEY
}

\author{
Banu ÇALIȘ USLU ${ }^{1}$ iD \\ ${ }^{1}$ Marmara University, Department of Industrial Engineering, 34722, Istanbul, TURKEY \\ *Corresponding author; bcalis@marmara.edu.tr
}

\begin{abstract}
Today, rapidly developing technological innovations affect and change all sectors. Health sectors are inevitably involved in a digital transformation. In this transformation, IoT technology plays an important role, which enables many devices to connect and work together. IoT converts systems into intelligent systems that work together using sensors, connection methods, internet protocols, databases, cloud computing and analytics as infrastructure. In this respect, it is necessary to establish the related technical infrastructure and generate a suitable environment for the establishment of intelligent hospitals. Finally, a capability model has been developed to measure the digital performance of Turkey. For Turkey health sector, the technology assessment report is presented based on survey results and HIMMS EMRAM data.
\end{abstract}

Keywords: Smart Hospital, Capability Model, HIMMS EMRAM

Received: January 5, $2020 \quad$ Accepted: March 16, 2020

\section{Introduction}

It has taken many years for the concept of IoT to emerge. With the increased computational power of devices, new methods and technologies allowed to be developed. The development and stages of digital computing over the years can be seen in Figure 1. With the rapid development, following the invention of the Turing Machine was described caused to use new methods of research and discovery that have emerged in the calculation methods used when working with a large data set. Digital computing used in today's computers gained importance in the 1950s with Alan Turing's intelligence computing machinery and intelligence abstract. One of the issues mentioned in the article is whether the machines can think and not play. In the 1960s, the cluster computing system came up. A computer network of multiple computers is a set of connected computing devices that work together in many respects. Each node in this network is designed to perform the same task through compatible software and is in a controllable and re-plannable form.

In the 1970s, the Stanford Artificial Intelligence Laboratory (SAIL) was established and continued with artificial intelligence and calculations. A knowledge-based appeared in the 1980s and definition of a complex software system that reasons and uses a knowledge base to solve complex and many different tasks. This knowledge-based explicit and reasoning system allows driving new technology. In the 1990s grid computing which is a method of distributed computing for dynamic and geographically determined 
in organizations comes from sharing computing, applications, network resources, and data warehousing. [1].

In the early 2000s, cloud computing, one of the foundations of IoT, has become an important issue. Cloud computing plays an important role in the unification of many resources. Cloud computing utilizes an easy, multiple, ad-hoc network permissions for configurable IT resources that can be quickly managed and released with minimal management effort or service provider interaction [2].

Then IoT based applications are becoming increasingly widespread and it is being forecasted that the IoT scenarios and applications will be increased to 20 billion units by 2020, up from 0.9 billion in 2009 $[3,4]$. Finally, fog computing is similar to cloud computing as the logic of work, but in terms of slow latency, location awareness, and improves quality-of-services (QoS) for streaming and real-time applications so digital computing takes a step further [5].

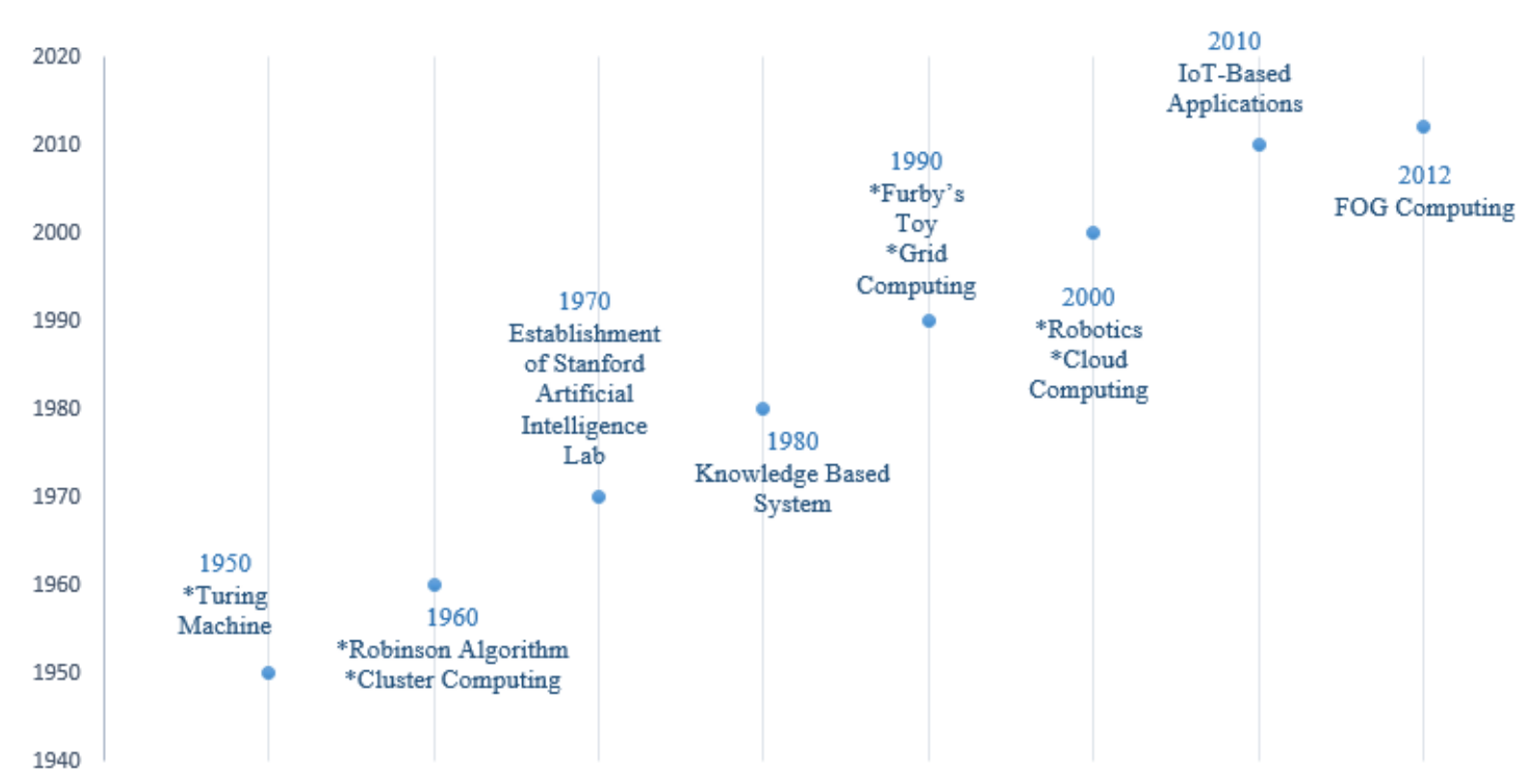

Figure 1. Digital computing and historical evolution

With these intelligent abilities, IoT technology enables information exchange between smart devices (things or objects) that satisfy a fast and accurate response to a particular problem that may occur in any of the system's stakeholders to improve Quality of Service (QoS). This information exchange is provided with continuous information flows between patients, doctors, medicine and biomedical suppliers, etc. so, IoT uses advanced IT technology to integrate the various components of a collaborative network to improve the efficiency, service capability, and flexibility between smart devices.

These smart devices can monitor and perceive their environmental conditions, measure the phenomena, the activities or the functions on the installed platforms, then convey the gathered data to a management unit/decision support system for further processing. Collected sensory data is used to understand the current situation of the system, providing information on the states of each unit in the network and the status of the complete system. and as a first step technology employs data processing technologies to transform raw data into input data. Processed input data is converted into meaningful 
information using information processing techniques and finally, this information is transformed into knowledge that enables the system to provide self-action through the knowledge processing approach without the involvement of humans [6]. In other words, IoT systems provide autonomous systems to control the complex system via self-governance and self-management abilities. [7].

\subsection{IoT-based smart hospital}

There are 7 main characteristics that need to be built in order to develop intelligent hospital applications based on IoT technology. These are; 1. Sensing, 2. Inter-Operability, 3. Communication, 4. Connection, 5. Networking, 6. Data Analytics, and 7. Artificial Intelligence (AI) capabilities. These characteristics are shown in Figure 2 from outside to inside.

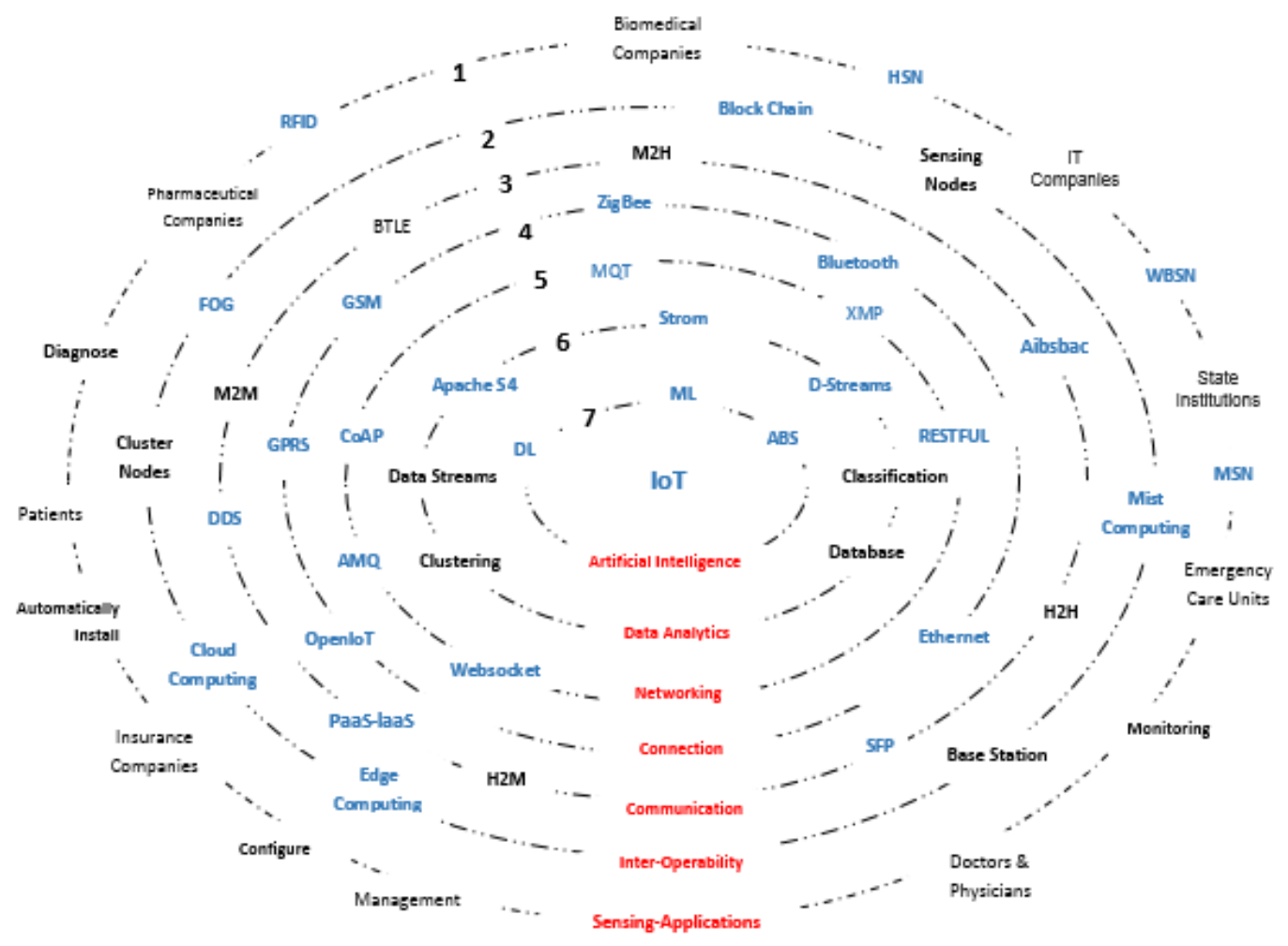

Figure 2. Seven Characteristics of IoT Technology for Smart Hospital

Sensing: Sensing capability defines the monitoring, storage, and analysis of health data by employing ubiquitous and distributed computing technology. Sensing characteristics of IoT technology define the data acquisition ability in real-time using low-energy to produce reliable messages [8].

Inter-operability: Inter-operability capability defines the ability to work with many heterogeneous devices at different scales simultaneously for a specific purpose. To build an interoperable system there is a strong need to define an efficient connection protocol and communication standard but lack of general standards leads to inefficient inter-operability [9].

Communication: IoT devices (things or objects) can communicate with each other within the same or heterogeneous networks [10]. This communication between things devices can be human to human $(\mathrm{H} 2 \mathrm{H})$, human to machine $(\mathrm{H} 2 \mathrm{M})$ or machine to machine $(\mathrm{M} 2 \mathrm{M})$ to analyze and produce information [11]. 
Connection: Connection capability defines to construct a secure channel between connected objects and base stations [12].

Network: IoT networking capability defines the selection of appropriate network protocol in order to build connections and communication between IoT devices [12].

Data Analytics - Artificial Intelligence: IoT technology undergone many changes in areas such as manual processing, mechanization, automation, information, integration, and intelligence. Especially, advances in the field of Robotics and AI (Artificial Intelligence) have emerged as key technologies in patient care and disease management [13]. Specifically, AI applications have made significant progress in personalized risk assessment, disease diagnosis and image processing for patients $[14,15]$. Also, the Integration of AI technologies and data analytics methods that including machine learning, game theory, optimization algorithms, and so on have enabled the creation of autonomous IoT structures that can self-repairing, self-healing, capable of protecting itself and self-organizing. And these capabilities have been used to make timely decisions to system needs [16] to a way of working is getting simpler and easier.

\section{Materials and Methods}

\subsection{Hospital capability model and evaluation of Turkey}

As a result of the digital transformation, significant changes have occurred in the health care processes of the hospitals and IoT technology has become one of the most important drivers of this change by enabling sensing, processing, and acting capability. In this rapid change, from data collection through intelligent devices to producing smart solutions there is a strong need to measure the level of digital capability of hospitals. In this chapter, the general structure of hospitals from data acquisition methods to data sharing processes was analyzed in accordance with the proposed infrastructure in chapter 2 and a capability model was created in this direction. This model aims to reveal the competency levels of the hospital in 3 levels and to identify the areas that are open to improvement in this direction. The proposed model is shown in Figure 3.

The first level is the basic level and it contains the basic characteristics of the initial level. At this level data only comes from clinical units and the local area network is used to transfer data through the system. Data collection and processing are done in central storage, cloud storage methods and systems are not used.

The second level is defined as integrated and contains partial stakeholder integration, machineto-machine communication between biomedical equipment, local area network and partial remote monitoring in addition to the basic characteristics. The common feature of the hospitals at this level is being chained hospital group and they can provide the integration with ERP program used in their structure. Data collection and processing are done in both central storage and cloud storage methods.

The third level is the level of the smart hospital that includes a low power area network that is integrated with both machine-to-machine, human-to-machine capabilities. Another important capability of the smart hospital level is the type of data collection. In this level, data come from not only clinical nodes but also patient-generated data and transportation data are possible to collect. In addition to network capability, a knowledge-based decision-based making system, which is composed of artificial intelligence and advanced analytical solutions, is also one of the most important properties of a smart hospital. 


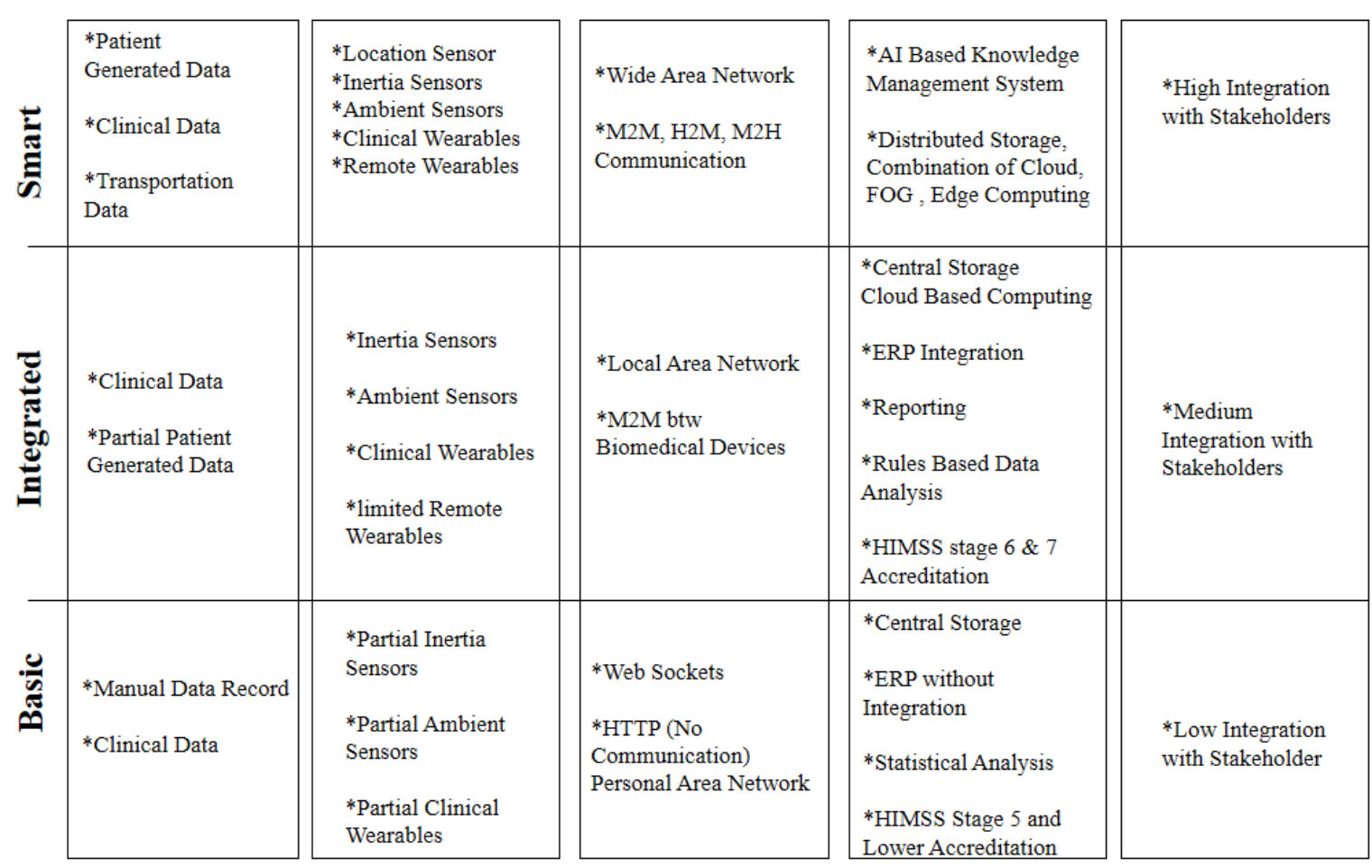

Figure 3. Hospital Digital Capability Model

\subsection{Capability Analysis Based on Survey}

Based on the proposed capability model, a questionnaire was designed to evaluate the devices, network and communication methods, system design parameters and data management methods required to create the infrastructure. Each problem was intensively used in the literature and a comprehensive list was created. Identified thanks to these questions turkey in a private hospital-tohospital survey applying the IoT system in terms of how much they have adequate infrastructure and development of open points that were examined.

Each question aims to obtain data on a different layer of the required technological infrastructure. Data pertaining to both the patient and the environment is recovered via sensors. In the questionnaire, the sensors were examined in 4 groups and defined as position sensors, wearable sensors, ambient sensors, and inertial sensors. Network systems are needed to integrate the data collected from the sensors with other systems, and different structures can understand each other through certain network protocols on these systems. Also, how often and how the data flow is done is an important issue because it plays a critical role in some scenarios and some cases create an additional system load and cost. While some of the questions are questioning whether to have an inventory or not, some questions have been determined directly to learn the design of the process and the methods used.

\subsection{Implementation of the survey}

Within the scope of the study, the survey was carried out with 5 different institutions in cooperation with its own IT department employees. 2 of these 5 hospitals are a group - chain hospitals, managed under a large holding. The other 2 are the research hospitals managed by the state institutions and units under the name of the university hospital. The remaining hospital is a single-branch private hospital managed by a company. 
Currently, the main reason why the survey is limited to 5 is that companies that have hospitals and manage them do not accept the survey. Institutional culture is not suitable for research, the technical infrastructure is not enough and they are hesitant to occur.

Also, companies with their private universities stated that they did not look favorably at research studies from different schools. Therefore, the number of surveys was kept at this level.

\section{Results}

Due to the lack of enough survey data, statistical analysis is not given in the report. Instead, the findings from the survey study are listed as follows.

- In the process of collecting data, RFID is mostly used technology to collect clinical data. It has been observed that the data about the follow-up of the patients can be collected on a real-time basis. However, there is no use of sensor technology during transport or remote patient followup.

- In order to satisfy an intelligent communication, there is a strong need for device integration. In this case, it is observed that M2M communication has been used between biomedical devices and reports obtained from this information exchange are accessible within the hospital information management system, however, these applications only involve in-hospital data exchange.

- Hospital systems were integrated with many institutions, so they did not record home health care, prescription and medical records in the digital environment.

- A wide area network infrastructure is not used when providing a personal area network and local area network for the establishment, connection, and connection of network connections.

- No hospital institution uses analytical methods using clinical and data collected from patients. Artificial intelligence, estimation, and detection methods are not used.

- In clinical studies, ambient sensors and sensors that can be attached to the patient are widely used. Magnetic field sensor is observed from inertial sensors while others are not used. The use of position sensors has not been observed. Patient and inventory monitoring cannot be performed smartly.

\subsection{Capability Analysis Based on HIMMS (Healthcare Information and Management Systems Society)-EMRAM (Electronic Medical Record Adaptation Model)}

In the health sector, measuring the digitalization level based on the specified standards is very important. EMRAM (Electronic Medical Record Adaptation Model) model is developed by HIMMS has proposed an important guide through a set of standards for the measuring of the digitalization process [17]; In this guide of HIMSS-EMRAM, the digitization levels of hospitals are divided into 7 stages. The properties defined for each stage are described below. 
Table 1. EMRAM stages [18].

\begin{tabular}{|c|c|}
\hline Stage & Definition \\
\hline 7 & $\begin{array}{l}\text { Measurement of electromagnetic radiation, data sharing with } \\
\text { different institutions, data analysis methods, ensuring business } \\
\text { continuity, privacy, and security }\end{array}$ \\
\hline 6 & $\begin{array}{l}\text { Breast milk validity, blood products, technological medication, and } \\
\text { risk reporting }\end{array}$ \\
\hline 5 & $\begin{array}{l}\text { Structured documentation for clinicians, fully clinical decision- } \\
\text { making systems, device security for attacks. }\end{array}$ \\
\hline 4 & $\begin{array}{l}\text { Ordering in the electronic environment, ensuring work processes } \\
\text { related to processes, documentation of nurses and staff }\end{array}$ \\
\hline 3 & Routine documentation and security of nurses and in-hospital staff \\
\hline 2 & $\begin{array}{l}\text { Storage of clinical data, cooperation with different institutions and } \\
\text { security of patient data at the basic level }\end{array}$ \\
\hline 1 & $\begin{array}{l}\text { Radiology measurement reports, laboratory test results, pharmacy } \\
\text { and drug records are installed in the system. }\end{array}$ \\
\hline 0 & $\begin{array}{l}\text { Most basic clinical work and medical systems are carried out } \\
\text { manually. }\end{array}$ \\
\hline
\end{tabular}

Stage 7, which is the top-level, is a zero paper environment. The characteristics of this stage are detailed below since it is the closest to the smart hospital structure at the clinical level;

- Patient registration, hospitalization, and other clinical procedures, consultations, and referrals are made from paper without a paper.

- E-prescription is used in the hospital.

- MR, X-ray, ECG, blood and other tests (hearing test, etc.) are done without any paper in a computer environment and the results of these requests are presented in a digital environment. Both health workers and patients can access these results via telephone and tablet.

- All data (records, results, invoices, etc.) are archived in a digital environment and information security is provided.

- Through the computer terminal in the patient rooms, nurses enter the system without any paperwork and paper, so that the pharmacy, inventory tracking and billing system can instantly record the outlets.

- In the closed-circuit drug system, the correct drug is administered at the right time with the right patient at the right dose.

\subsection{Results base on the analysis of HIMSS-EMRAM Accreditation}

T.C. Ministry of Health has adopted the use of HIMSS-EMRAM accreditation as a strategic decision to measure digital conversion levels of hospitals in Turkey [18]. However, in accordance with the information accessed from HIMMS's site, it was seen that there were only 2 hospitals that reached stage 7 in Turkey [17]. These are Tire State Hospital and Yozgat City Hospital.

The distribution of the countries who have stage 7 accreditation in Europe is shown in Figure 4. 


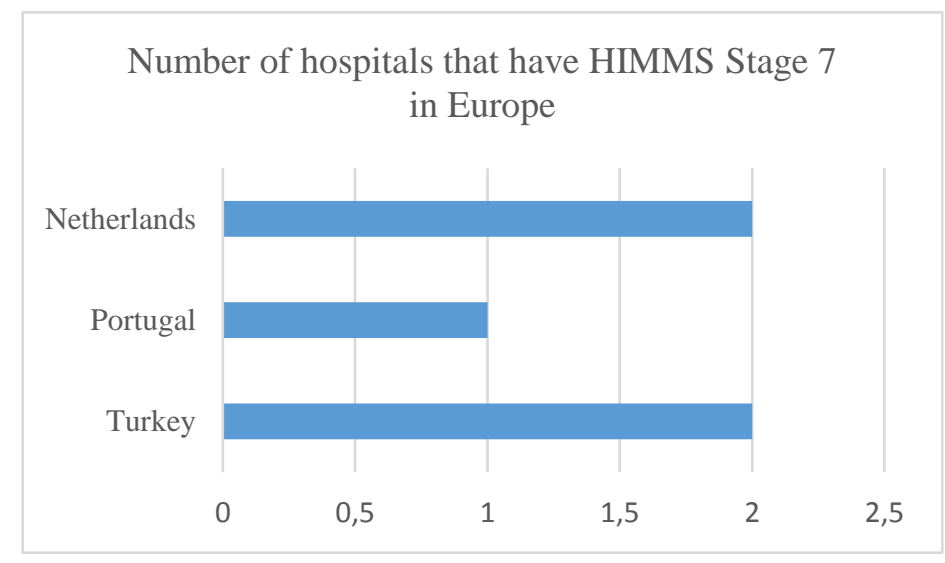

Figure 4. Distribution of Stage 7 accredited hospitals in Europe

In the analysis performed based on Step 6, it is seen that 163 accredited hospitals exist in Turkey. The distribution of the sixth stage accredited countries in Europe is shown in Figure 5.

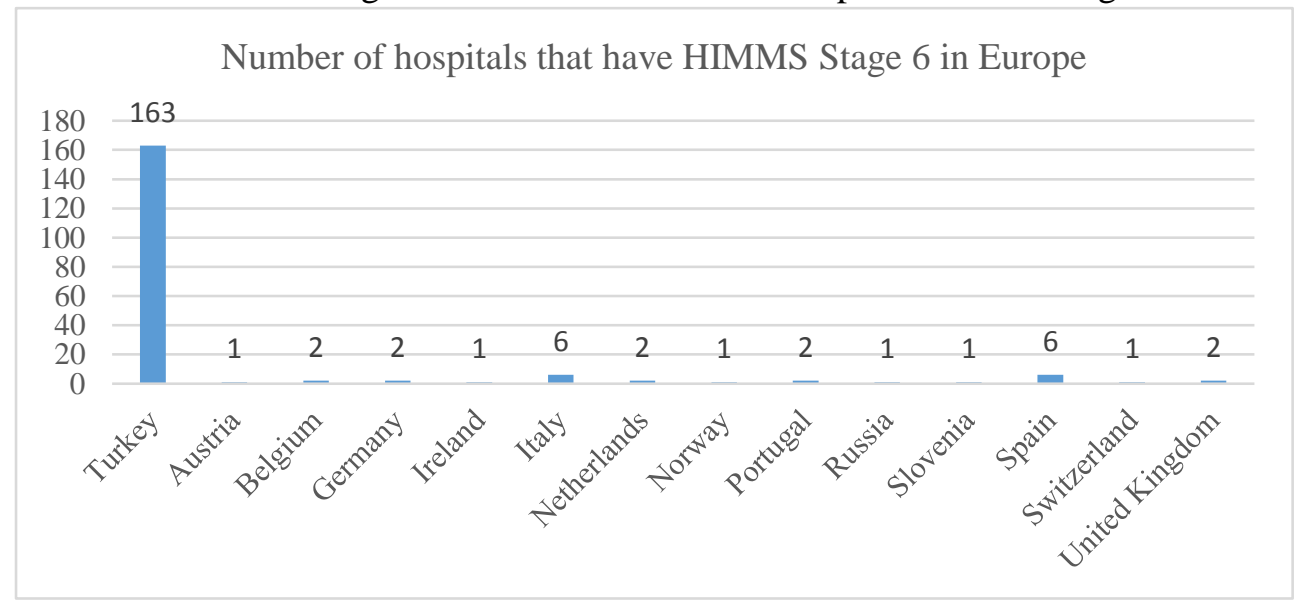

Figure 5. Distribution of Stage 6 accredited hospitals in Europe

According to data from the Ministry of Health, the number of inpatient health institutions in Turkey is approximately 1518 [19]. Considering the number of hospitals that have Stage 6 or 7, It is very clearly seen that the process of digital transformation needs to improve in many ways in Turkey. Only $0.13 \%$ of the hospitals are at the zero paper level. In addition, only $10.7 \%$ of the total number of hospitals were accredited by providing 50\% digitalization in the processing of data in the clinical process.

\section{Conclusions}

In this study, firstly, the literature review has been done to analyze the current studies and characteristics of the IoT technology is determined. Then a questionnaire is prepared to measure the existing competencies of institutions and results are analyzed. In addition to survey results HIMMSEMRAM digital accreditation is evaluated to analyze the capability of hospitals in Turkey. The study will be taken forward to increase the effect of IoT technology on the hospital. Findings from the project are summarized below. 
I. In the process of gathering clinical data, some areas can be seriously improved in the collection and integration of data from patients.

II. In the infrastructure technology used for the integration of the data, it has been observed that the network area and local area network is used intensively, and it is seen that the necessary infrastructure works are not enough to develop the infrastructure especially in the wide-area network and low power area network systems.

III. It has been determined that the decision support systems are especially effective in the followup of patients by nurses, but they are important areas for the development of disease detection.

IV. It has been determined that patient intervention processes are prolonged due to lack of system integrations for data of patients in ambulance vehicles such as ambulance used in patient transportation and transportation stages and it is very clear to improve in this area.

V. It was determined that the integration with other stakeholders was the strongest infrastructure in terms of the IoT infrastructure and that integration with the pharmaceutical and biomedical firms was open to improvement.

VI. It has been determined that labor force planning can be carried out depending on the legislation, however, dynamic density is not sufficient to measure simultaneous workforce planning and there is a need for improvement in this area.

Future works within the scope of this study are it is aimed to digitize the capability model developed for the IoT-based intelligent hospital design, to present it as an open-source, and to design a more comprehensive project to obtain the wider analysis.

The compliance to the Research and Publication Ethics: This study was carried out in accordance with the rules of research and publication ethics.

Ethical Process: Ethics committee approval is not required for this study.

\section{References}

[1] Foster I., Kesselmann C., The Grid: Blueprint for a New Computing Infrastructure, Morgan Kaufmann Publishers, Chicago, 1999.

[2] Hashemi, S. M., \& Bardsiri, A. K., "Cloud computing vs. grid computing”, ARPN Journal of systems and software, 2(5), 188-194, 2012.

[3] Lee, I., \& Lee, K., "The Internet of Things (IoT): Applications, investments, and challenges for enterprises", Business Horizons, 58(4), 431-440, 2015.

[4] Uslu, B. Ç., \& Fırat, S. Ü., "A Comprehensive Study on Internet of Things Based on Key Artificial Intelligence Technologies and Industry 4.0", Advanced Metaheuristic Methods in Big Data Retrieval and Analytics. IGI Global, Oran, (pp. 1-26). 2019

[5] Stojmenovic, I., \& Wen, S., "The fog computing paradigm: Scenarios and security issues. In Computer Science and Information Systems (FedCSIS)", Federated Conference on 2014, (pp. 1-8). IEEE, 2014.

[6] Al-Fuqaha, A., Guizani, M., Mohammadi, M., Aledhari, M., \& Ayyash, M., "Internet of things: A survey on enabling technologies, protocols, and applications", IEEE Communications Surveys \& Tutorials, 17(4), 2347-2376, 2015. 
[7] Sterritt, R., \& Hinchey, M. (2005, April). "Why computer-based systems should be autonomic"?, 12th IEEE International Conference and Workshops on the Engineering of Computer-Based Systems (ECBS'05, Greenbelt, MD, USA, 2005, (pp. 406-412).

[8] Wan, J., Zou, C., Zhou, K., Lu, R., \& Li, D., "IoT sensing framework with inter-cloud computing capability in vehicular networking”, Electronic Commerce Research, 14(3), 389-416, 2014.

[9] Aloi, G., Caliciuri, G., Fortino, G., Gravina, R., Pace, P., Russo, W., \& Savaglio, C., "Enabling IoT interoperability through opportunistic smartphone-based mobile gateways", Journal of Network and Computer Applications, 81, 74-84, 2017.

[10] Bello, O., \& Zeadally, S., "Communication Issues in the Internet of Things (IoT)", Next-Generation Wireless Technologies (pp. 189-219). Springer, London, 2013.

[11] Madakam, S., Ramaswamy, R., \& Tripathi, S., "Internet of Things (IoT): A literature review", Journal of Computer and Communications, 3(05), 164, 2015.

[12] Naik, N., "Choice of effective messaging protocols for IoT systems: MQTT, CoAP, AMQP and HTTP”, 2017 IEEE international systems engineering symposium (ISSE), Vienna, Austria, 2017, (pp. 1-7).

[13] Tsang, L., Kracov, D. A., Mulryne, J., Strom, L., Perkins, N., Dickinson, R., et al., "The impact of artificial intelligence on medical innovation in the European Union and united states", Intellectual Property \& Technology Law Journal, 29(8), 3-11, 2017.

[14] Keleş, A., \& Yavuz, U., "Expert system based on neuro-fuzzy rules for diagnosis breast cancer". Expert Systems with Applications, 38(5), 5719-5726, 2011.

[15] Paydar, S., Pourahmad, S., Azad, M., Bolandparvaz, S., Taheri, R., Ghahramani, Z., ... \& Abbasi, H. R., "The Evolution of a Malignancy Risk Prediction Model for Thyroid Nodules Using the Artificial Neural Network", Middle East Journal of Cancer, 7(1), 47-52, 2015.

[16] Krupitzer, C., Roth, F. M., VanSyckel, S., Schiele, G., \& Becker, C., “A survey on engineering approaches for self-adaptive systems", Pervasive and Mobile Computing, 17, 184-206, 2015.

[17] HIMMS, "Healthcare Information and Management System Society", HIMMS EMRAN STAGE6\&7 COMMUNITY, https://www.himss.eu/communities/himss-emram-stage-6-7community, 2019.

[18] About EMRAM, Ministry of Health Digital Hospital website, https://dijitalhastane.saglik.gov.tr/TR,4858/emram-hakkinda.html, 2019

[19] Number of Physicians, Number of Persons per Physicians and Number of Patient Hospital Visits per Physician, TUIK, Turkish Statistical Institute, www.turkstat.gov.tr , 2017. 